\title{
A time-domain SNR estimator based on a periodic preamble for wireless OFDM systems
}

\author{
Sekchin Chang ${ }^{1 \mathrm{a})}$ and Brian Kelley ${ }^{2}$ \\ ${ }^{1}$ School of Electrical and Computer Engineering, The University of Seoul, Seoul, \\ Korea \\ ${ }^{2}$ Department of Electrical and Computer Engineering, The University of Texas at \\ San Antonio, San Antonio, Texas, USA \\ a) schang213@uos.ac.kr
}

\begin{abstract}
A novel SNR estimator is presented for efficient estimation of SNR in wireless OFDM systems. Unlike the conventional SNR estimators, the proposed estimator calculates an SNR estimate in the time domain, which alleviates the usage of FFT. In addition, the SNR estimator effectively exploits a periodic feature of the OFDM preamble for accurate SNR estimation. Furthermore, the SNR estimator shows robustness to timing-offset. The simulation results indicate that the presented estimator is superior to the conventional SNR estimators in terms of NMSE in the case of imperfect synchronization under high frequency-selectivity channel.
\end{abstract}

Keywords: SNR estimator, preamble, OFDM

Classification: Wireless communication hardware

\section{References}

[1] A. Ghosh, D. R. Wolter, J. G. Andrews, and R. Chen, "Broadband wireless access with WiMax/802.16: current performance benchmarks and future potential," IEEE Commun. Mag., vol. 43, no. 2, pp. 129-136, Feb. 2005.

[2] A. Goldsmith, "Wireless Communications," Cambridge, 2006.

[3] S. Boumard, "Novel noise variance and SNR estimation algorithm for wireless MIMO OFDM systems," Proc. IEEE GLOBECOM, vol. 3, pp. 13301334, Dec. 2003.

[4] C. Seo, I. Cho, S. Portugal, J. Park, and G. Park, "Performance analysis of a new preamble-based SNR estimation algorithm," IEICE Trans. Commun., vol. e94-b, no. 5, pp. 1498-1501, May 2011.

[5] M. Zivkovic and R. Mathar, "Preamble-based SNR estimation in frequency selective channels for wireless OFDM systems," Proc. IEEE VTC Spring, pp. 1-5, 2009.

[6] C. W. Therrien, Discrete Random Signals and Statistical Signal Processing, Prentice-Hall, 1992.

[7] Joint Technical Commiteee of Committee T1 R1P1.4 and TIA TR46.3.3/TR45.4.4 on Wireless Access, "Draft Final Report on RF Channel Characterization," Paper No. JTC(AIR)/94.0.1.17-238R4, Jan. 17, 1994. 


\section{Introduction}

Currently, orthogonal frequency division multiplexing (OFDM) is a key modulation scheme to enable broadband cellular systems such as WiMax [1]. Especially, the latest OFDM-based wireless systems exploit adaptive modulation and coding (AMC) [2] and adaptive subcarrier allocation schemes in order to increase the system capacity. Since the schemes usually utilize the SNR value of the received signal, an accurate SNR estimation is required in the OFDM-based wireless systems. Several SNR estimators have been proposed in OFDM wireless systems $[3,4,5]$. For SNR estimation, the conventional approaches exploit a preamble which is usually employed in OFDM systems for synchronization and channel estimation. The conventional methods estimate the SNR value of the received signal using the preamble in the frequency domain. Therefore, the methods require FFT usage and perfect synchronization.

In this letter, a novel SNR estimator is proposed for efficient and accurate SNR estimation in OFDM wireless systems. The proposed estimator calculates the SNR value of the received signal in the time domain, which lessens the overhead of the FFT usage. In addition, for accurate SNR estimation the proposed estimator effectively exploits a periodic feature of the presented preamble which consists of several identical blocks. Moreover, the suggested estimator exhibits insensitivity to timing-offset while the conventional estimators are very sensitive to timing-offset. The simulation results show that the proposed SNR estimator has better performance than the conventional SNR estimators in terms of normalized mean squared error (NMSE) with the same overhead of preambles.

\section{The conventional SNR estimators}

Fig. 1 illustrates the architecture and the preamble for the conventional SNR estimators such as Boumard's SNR estimator [3] and Seo's SNR estimator [4]. As shown in Fig. 1 (a), the estimators rely on FFT module in order to estimate signal and noise powers in the frequency domain. In addition, the SNR estimators exploit the preamble of Fig. 1 (b) for SNR estimation. The preamble consists of two identical symbols and the length of each preamble symbol is equivalent to that of OFDM symbol. After FFT, the received signal $Y(i, k)$ of Fig. 1 (a) can be expressed as

$$
Y(i, k)=C(i, k) H(i, k)+\Upsilon(i, k)
$$

where $C(i, k), H(i, k)$, and $\Upsilon(i, k)$ denote the transmitted data symbol, the channel frequency response, and additive white Gaussian noise (AWGN) on the $k$ th subcarrier in the $i$ th preamble symbol, respectively. In (1), $|C(i, k)|^{2}=1$ since OFDM standards usually adopt preambles which consist of QPSK or BSPK modulated subcarriers. In addition, $C(1, k)=C(2, k)=$ $C(k)$ since the preamble symbols are identical, and $H(1, k)=H(2, k)=H(k)$ since the channel is assumed to be time-invariant during the SNR estimation on the preamble symbols. In the conventional SNR estimators, perfect 


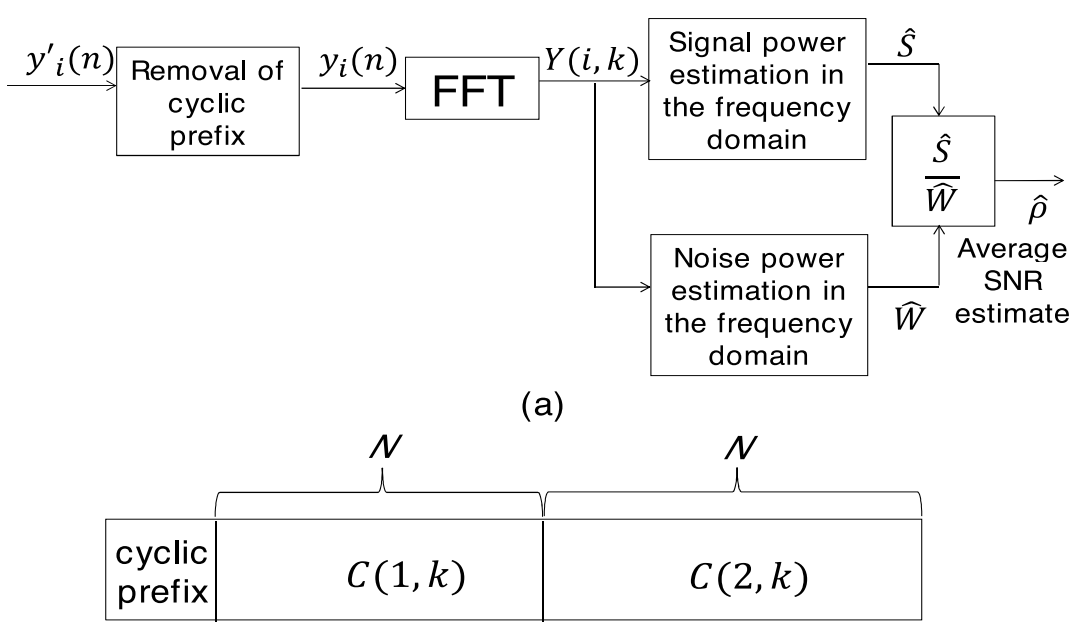

(b)

Fig. 1. The architecture and the preamble for the conventional SNR estimators

synchronization is assumed since the frequency-domain signal of (1) is utilized $[3,4,5]$.

\subsection{Boumard's SNR estimator}

Boumard originally proposed an SNR estimation scheme for $2 \times 2$ MIMOOFDM systems assuming that the channel varies slowly in both time and frequency domains [3]. The corresponding SNR estimation algorithm can be found in [5] for SISO-OFDM systems. In Boumard algorithm, the average SNR estimate $\hat{\rho}_{\text {Bou }}$ is calculated as

$$
\hat{\rho}_{\text {Bou }}=\frac{\hat{S}_{\text {Bou }}}{\hat{W}_{\text {Bou }}}
$$

where $\hat{S}_{\text {Bou }}$ and $\hat{W}_{\text {Bou }}$ denote the signal power estimate and the noise power estimate, respectively. In (2), $\hat{S}_{\text {Bou }}$ is achieved as follows:

$$
\hat{S}_{\text {Bou }}=\frac{1}{N} \sum_{k=0}^{N-1}|\hat{H}(k)|^{2}
$$

where $N$ is the size of FFT. In $(3), \hat{H}(k)$ can be calculated using

$$
\hat{H}(k)=\frac{C^{*}(k)}{2}[Y(1, k)+Y(2, k)]
$$

In (2), $\hat{W}_{\text {Bоu }}$ is achieved as follows:

$$
\begin{aligned}
\hat{W}_{\text {Bou }}=\frac{1}{4 N} \sum_{k=1}^{N-1} & \mid C(k-1)[Y(1, k)+Y(2, k)] \\
& -\left.C(k)[Y(1, k-1)+Y(2, k-1)]\right|^{2}
\end{aligned}
$$

The estimation of (5) is valid when the channel varies slowly in the frequency domain. Therefore, the estimation of (5) exhibits high sensitivity to frequency selectivity. Since the estimation must be calculated after FFT in (4) and (5), a timing-offset may cause an inaccurate estimate. 


\subsection{Seo's SNR estimator}

Seo's SNR estimator [4] exhibits robustness to the frequency selectivity by calculating the noise power estimate $\hat{W}_{\text {Seo }}$ as follows:

$$
\hat{W}_{\text {Seo }}=\frac{1}{N} \sum_{k=0}^{N-1}|Y(1, k)-Y(2, k)|^{2}
$$

Since the estimation must be calculated after FFT in (6), a timing-offset may still cause an inaccurate estimate.

\section{The time-domain SNR estimator based on a periodic pream- ble}

Fig. 2 illustrates the architecture and the preamble for the proposed SNR estimator. Since the estimation is performed in the time domain, the estimator does not rely on FFT module as shown in Fig. 2 (a). The proposed estimation utilizes the periodic preamble which consists of $M$ identical blocks as shown in Fig. 2 (b). In the preamble, each block is composed of $q$ samples. After the removal of the cyclic prefix, the received signal $y(n)$ of Fig. 2 (a) can be expressed as

$$
y(n)=h(n) \otimes c(n)+v(n), \quad n=0,1, \ldots, q M-1
$$

where $\otimes$ denotes a circular convolution, and $c(n)$ and $v(n)$ represent the transmitted preamble data and AWGN in the time domain, respectively. In $(7)$, let $h(n) \otimes c(n)$ be $c_{h}(n)$ which is the transmitted preamble data affected by the time-domain channel $h(n)$. If the channel parameter $h(n)$ keeps constant during the SNR estimation on the preamble, the $c_{h}(n)$ is repeated every $q$ samples due to the periodic feature of the preamble blocks. Therefore, $y(n)$ of $(7)$ can also be expressed as

$$
y(n)=c_{h}(n \bmod q)+v(n), \quad n=0,1, \ldots, q M-1
$$

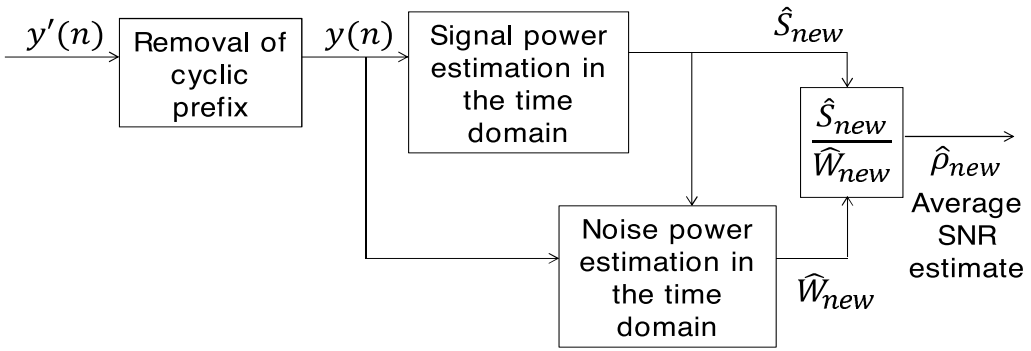

(a)

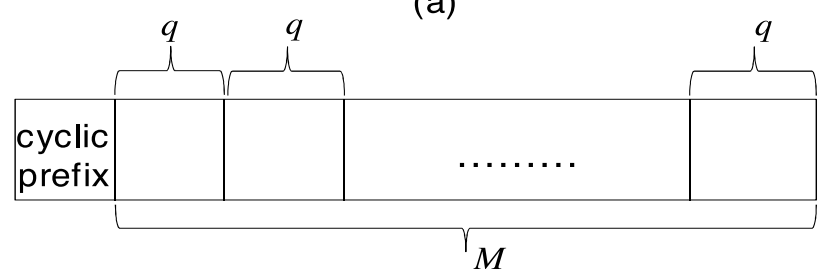

(b)

Fig. 2. The architecture and the preamble for the proposed SNR estimator 
Since $c_{h}(n \bmod q)$ and $v(n)$ are uncorrelated, $E[v(n)]=0$, and $E\left[v(l) v^{*}(m)\right]=0, l \neq m$ in (8), the correlation of $y(n)$ and $y(n+q)$ can be expressed as

$$
\begin{aligned}
\mathrm{R}_{y} & =E\left[y(n+q) y^{*}(n)\right] \\
& =E\left[\left|c_{h}(n \bmod q)\right|^{2}\right]=S_{\text {new }}
\end{aligned}
$$

where $S_{\text {new }}$ indicates the signal power, and $E[\cdot]$ denotes the expectation operator. From (9), the signal power estimate $\hat{S}_{\text {new }}$ can be calculated in the time domain as follows:

$$
\hat{S}_{\text {new }}=\frac{1}{q \cdot(M-2)} \sum_{n=0}^{q \cdot(M-2)-1} y(n+q+p) y^{*}(n+p)
$$

where $p$ denotes a timing-offset. If $-q+L \leq p \leq q$ in (10) where $L$ is a channel length which is related with delay spread, the signal estimate $\hat{S}_{\text {new }}$ is valid due to the cyclic prefix and the last preamble block even in the case of imperfect synchronization. This is feasible since the timing-offset usually ranges on a few-sample interval after imperfect synchronization. Using the uncorrelated property between $c_{h}(n \bmod q)$ and $v(n)$, and $E[v(n)]=0$ in (8), the total power of the received signal $y(n)$ can be written as

$$
\begin{aligned}
\mathrm{P}_{y} & =E\left[|y(n)|^{2}\right] \\
& =E\left[\left|c_{h}(n \bmod q)\right|^{2}\right]+E\left[|v(n)|^{2}\right] \\
& =S_{\text {new }}+W_{\text {new }}
\end{aligned}
$$

where $W_{\text {new }}$ indicates the noise power. From (11), the total power estimate $\hat{\mathrm{P}}_{y}$ can be calculated in the time domain as follows:

$$
\hat{\mathrm{P}}_{y}=\frac{1}{q \cdot(M-2)} \sum_{n=0}^{q \cdot(M-2)-1}|y(n+p)|^{2}
$$

where $p$ denotes a timing-offset. If $-q+L \leq p \leq q$ in (12) where $L$ is a channel length, the total power estimate $\hat{\mathrm{P}}_{y}$ is also valid due to the cyclic prefix and the last preamble block even in the case of imperfect synchronization. This is still feasible as stated earlier. From (10), (11), and (12), the noise power estimate $\hat{W}_{\text {new }}$ can be estimated as follows:

$$
\hat{W}_{\text {new }}=\hat{\mathrm{P}}_{y}-\hat{S}_{\text {new }}
$$

Finally, the average SNR estimate $\hat{\rho}_{\text {new }}$ can be achieved using

$$
\hat{\rho}_{\text {new }}=\frac{\hat{S}_{\text {new }}}{\hat{W}_{\text {new }}}
$$

Note that the preamble of Fig. 2 (b) can also be exploited for channel estimation in the time domain. The least squares (LS) method [6] can be considered the time-domain channel estimation scheme which is widely utilized in various wireless systems including GSM cellular systems. The time-domain 
channel estimates can easily be converted into the corresponding frequencydomain channel estimates using FFT in OFDM systems. Though the LS scheme usually shows higher computational complexity than the frequencydomain channel estimation, the LS-based channel estimation produces more accurate channel estimates with the preamble of Fig. 2 (b) than the frequencydomain channel estimation with the preamble of Fig. 1 (b) since more blocks of Fig. 2 (b) can further suppress the AWGN effects.

\section{Simulation result}

Simulation results exhibit the effectiveness of the proposed SNR estimator for wireless OFDM systems. In this simulation, one OFDM symbol consists of 64 subcarriers. Therefore, $N=64$ in (3), (5), and (6). The length of cyclic prefix is 16 in Figs. 1 (b) and 2 (b). In Fig. 2(b), the block size $(q)$ and the number of blocks $(M)$ are 16 and 8, respectively. Therefore, the conventional SNR estimator and the proposed SNR estimator have the same preamble overhead (128 samples). It is assumed in the simulation that the OFDM system operates at $2.4 \mathrm{GHz}$ carrier frequency and occupies $20 \mathrm{MHz}$ bandwidth. In addition, the JTC indoor office B [7] is considered the frequency-selective channel in this simulation. In the OFDM system, the channel length $(L)$ of the JTC indoor office B is 15 , which indicates that the channel has high frequency selectivity. The performance for the SNR estimators is evaluated in terms of NMSE which is defined as

$$
\mathrm{NMSE}=\frac{1}{N_{t}} \sum_{i=1}^{N_{t}}\left(\frac{\hat{\rho}_{a v, i}-\rho_{a v}}{\rho_{a v}}\right)^{2}
$$

where $N_{t}$ is the number of independent trials $(50,000), \hat{\rho}_{a v, i}$ is the average SNR estimate in the $i$ th trial, and $\rho_{a v}$ is the actual SNR value.

Fig. $3(\mathrm{a})$ and (b) show the NMSE performance for SNR estimators in the case that the timing-offset $(p)$ is 0 and the timing-offset $(p)$ is 4 , respectively. As shown in Fig. 3 (a), the Boumard's estimator exhibits the worst NMSE performance since the estimator is sensitive to high frequency selectivity which exists in the JTC indoor office B channel. The proposed and the Seo's SNR estimators show very low NMSE values in the entire SNR range of Fig. 3 (a) in the case of high frequency selectivity. Especially, both SNR estimators exhibit almost identical NMSE performance when perfect synchronization $(p=0)$ is assumed as shown in Fig. $3(\mathrm{a})$. However, when the timing-offset $(p)$ is 4 in Fig. $3(\mathrm{~b})$, the Bourmard's and the Seo's estimators show significant degradations in the NMSE performance since the estimators utilize the frequency-domain signals after FFT. On the other hand, the proposed SNR estimator is robust to the timing-offset as exhibited in Fig. 3 (b).

\section{Conclusion}

A time-domain SNR estimator is suggested in this letter for efficient SNR estimation in wireless OFDM systems. The estimator alleviates the overhead of FFT usage unlike the conventional SNR estimators since SNR estimation 



Fig. 3. The NMSE performance for SNR estimators in the case that (a) the timing-offset $(p)$ is 0 and (b) the timing-offset $(p)$ is 4

is performed in the time domain. The proposed estimator relies on a periodic preamble in order to acquire an accurate SNR estimate. Simulation results show that the proposed SNR estimator outperforms the conventional SNR estimators with the same overhead of preambles. When perfect synchronization is assumed, the presented SNR estimator exhibits much better NMSE performance than Boumard's estimator, and shows NMSE performance comparable to Seo's estimator in the case of high frequency-selectivity channel. Moreover, the proposed SNR estimator is much superior to the conventional SNR estimators in terms of NMSE in the case of imperfect synchronization under high frequency-selectivity channel.

\section{Acknowledgments}

This work was supported by National Research Foundation of Korea Grant funded by the Korean Government (2011-0026962). 